\title{
Density anomalies and reentrant spinodal behavior th
}

\author{
Srikanth Sastry a, Francesco Sciortino ${ }^{\mathrm{a}, \mathrm{b}}$ and H. Eugene Stanley ${ }^{\mathrm{a}}$ \\ - Center for Polymer Studies and Department of Physics, Boston University, Boston, MA 02215, USA \\ - CRS4, Centro di Ricerca, Sviluppo e Studi Superiori in Sardegna, P.O. Box 488, 09100 Cagliari, Italy
}

Received 8 February 1993; in final form 16 March 1993

\begin{abstract}
A lattice model that displays anomalous density behavior and exhibits a reentrant spinodal is developed. This model is solved in the mean field approximation and the interrelation between density maxima and the reentrance of the spinodal is explicitly demonstrated. The limits of stability of the liquid phase are obtained at positive as well as negative pressures, and the nature of the limits of stability clarified. The interplay between local energy and local entropy is found to control the thermodynamics of the system.
\end{abstract}

Considerable effort has been devoted in recent years to the study of thermodynamic properties of liquids exhibiting density anomalies, with an emphasis on behavior in the metastable state and the limits of stability. Water is the best known of such liquids; it is also a well known example of a liquid with highly directional interactions, the hydrogen bonds [1-3].

Lattice-gas or Ising models with temperature-dependent interactions and fields have been used to model physical systems where entropic effects arising from local geometries play a significant role. Such entropic effects arise typically in systems with directional bonding interactions between constituents, which can take place only when the local geometric arrangement is favorable. These interactions lead to a non-trivial relationship between the system temperature and the temperature as measured by the effective interactions between constituents, reflected in the behavior of the interaction strengths defining the model. Such models with temperature dependent interactions have proven successful in describing many systems with orientation dependent interactions, like polymer solutions and binary mixtures with lower critical solution temperatures [4].

In this Letter, we introduce a lattice-gas model as a step towards understanding the microscopic mech-

\footnotetext{
* This work is based in part on the Ph.D. Thesis of S. Sastry.
}

anisms underlying the interplay between anomalous density behavior and the limits of stability of the liquid phase in systems where orientational degrees of freedom play a significant role. We define the model in terms of an effective interaction that is temperature dependent, and discuss the motivation for such a definition. The model exhibits phases that can be identified with gas, liquid and a low density solid. We obtain a liquid-gas limit of stability (spinodal) that is reentrant and reaches positive pressures at both the high and low temperature ends. We show that at low temperatures the liquid-gas spinodal meets the liquid-solid spinodal tangentially, and gives way to the liquid-solid spinodal, which becomes the physically relevant limit of stability.

The system we wish to describe has strong directional interactions between molecules which define a low temperature solid phase characterized by an open structure. States with higher local densities in the liquid phase are characterized by an increase in energy and orientational entropy, since interactions where no strong bonding takes place can occur in many orientational states.

To define a lattice model which incorporates these features, we consider a bcc lattice, which is divided into two interpenetrating diamond sublattices $A$ and B. On each site $i$, we define an occupancy variable $n_{i}=0,1$. The complete occupation of one of the sublattices with the other sublattice empty defines the 
low density open structure of the solid. Whether the occupation of sites on the other sublattice is favorable or not is determined by the competition between the interaction energy (which disfavors such occupation) and orientational entropy (which favors it). The strong interactions are dircctional, and hence can occur only for specific orientations. For other orientations, the interactions are weaker. At high $T$, the entropically favorable weak interactions are dominant in determining the effective interactions, while as $T$ is lowcred, the encrgctically favorable directional interactions become dominant. Thus, as $T$ is varied, there is an effective change in the interaction. We define the lattice-gas model by summing over the orientational degrees of freedom in a detailed Hamiltonian [4,5]. The interaction energy between sites on different sublattices then becomes a function of temperature, $J_{1} \equiv J_{1}(T)$. The interaction between site occupancy variables $n_{i}$ is thus the "internal free energy" of molecules arising from the orientational degrees of freedom. The resulting Hamiltonian is

$$
\begin{aligned}
\mathscr{H} & =-2 J_{1}(T) \sum_{\langle i j\rangle}^{(\mathrm{AB})} n_{i} n_{j} \\
& -2 J_{2} \sum_{\langle i k\rangle}^{(\mathrm{AA})} n_{i} n_{k}-\mu \sum_{i} n_{i} .
\end{aligned}
$$

Here

$$
\begin{aligned}
& J_{1}(T) \equiv 2 \epsilon \\
& -k_{\mathrm{B}} T \ln \left(1+\frac{1}{q}\left[\exp \left(2 J / k_{\mathrm{B}} T\right)-1\right]\right)
\end{aligned}
$$

is the $\mathrm{AB}$ interaction strength, $J_{2}=2 \mathrm{\epsilon}$ is the AA interaction strength, and $\mu$ is the chemical potential. In (2), $\epsilon$ corresponds to the "normal liquid" interaction between non-bonding AB pairs, $q$ is the number of orientational states, and $J$ corresponds to the energy cost of occupying adjacent $A B$ pairs when the molecules are properly oriented for directional interactions. Directional interactions can occur only between AA pairs and hence the interaction between $A B$ pairs results in their disruption. There are fewer orientations where directional interactions are possible than those where directional interactions are not possible. Hence, at high $T$, where entropic effects dominate, $J_{1}(T)$ approaches $2(\epsilon-J / q)$. As $T$ de- creases, $J_{1}(T)$ moves away from this value, eventually crossing over to the opposite sign and approaching $2(\epsilon-J)$ as $T$ approaches zero. We denote by $T_{\mathrm{tr}}$ the temperature where $J_{1}(T)$ is zero. The variation of $J_{1}(T)$ with temperature is shown in fig. 1a.

To solve the modcl defincd by (1) in the mean field approximation, we first express the grand potential $\Omega$ in terms of two density variables $\left(n_{\mathrm{A}}, n_{\mathrm{B}}\right)$, corresponding to the two sublattices. Above $T_{\mathrm{tr}}$, where both $J_{1}$ and $J_{2}$ are positive, the equilibrium solutions yield $n_{\mathrm{A}}=n_{\mathrm{B}}$. Below $T_{\mathrm{tr}}$, the first neighbor coupling $J_{1}$ changes sign, so that we have repulsive $\mathrm{AB}$ and attractive $\mathrm{AA}$ interactions. In the Ising model mapping of the lattice gas, these interactions define a metamagnet. Two new minima appear in the grand potential below $T_{\mathrm{tr}}$, associated with the ordering on one sublattice (i.e. $n_{\mathrm{A}}>\frac{1}{2}, n_{\mathrm{B}}<\frac{1}{2}$ and vice versa). We shall refer to this low density ordered phase as the solid phase.

The equilibrium phase boundaries between the
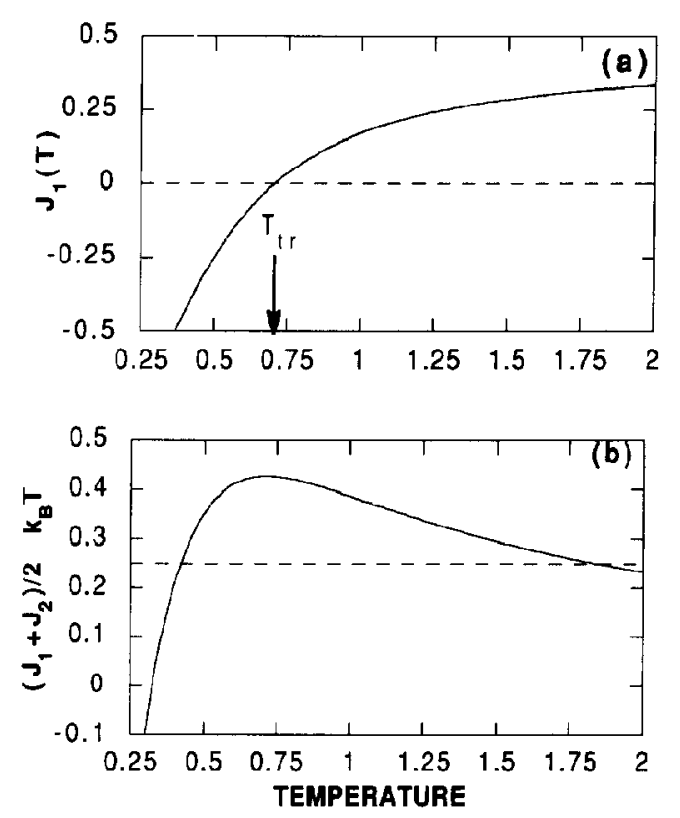

Fig. 1. (a) The effective $A B$ interaction strength as a function of temperature (solid line) for $\epsilon=0.3, J=1.0$ and $q=12$. The temperature and the effective interaction are expressed in units of $J$, i.e. by fixing $k_{\mathrm{B}}=1$. (b) $\left(J_{1}+J_{2}\right) / 2 k_{\mathrm{B}} T$ as a function of temperature (solid line). Also shown is the value of $1 / z$ (dashed line). The two points of intersection correspond to critical points for liquid-gas coexistence. 
solid and the liquid/gas phases are found by comparing the grand potential for the respective solutions, the phase boundary being given by the condition that the grand potential $\Omega$ for the two phases be the same. We obtain three coexistence lines, one between the upper critical point for the liquid-gas transition and $T_{\mathrm{tr}}$, and two coexistence lines between solid and liquid or gas phases respectively. These three coexistence lines meet at $T_{\mathrm{tr}}$. Since at $T_{\mathrm{tr}}$ we have three-phase coexistence, we identify $\left(T_{\mathrm{tr}}, P_{\mathrm{tr}}\right)$ as the triple point. Fig. 2 shows the coexistence lines in the $P-T$ plane. Note that the liquid-solid coexistence line has negative slope, as observed in water at lower pressure.

We next focus on the liquid phase and its limits of stability. We find the equation of state for the liquid and gas phase $\left(n_{\mathrm{A}}=n_{\mathrm{B}}=n_{\mathrm{eq}}\right)$ to be

$P_{\mathrm{eq}}=-z\left(J_{1}+J_{2}\right) n_{\mathrm{eq}}^{2}-k_{\mathrm{B}} T \ln \left(1-n_{\mathrm{eq}}\right)$.

From the equation of state, we locate the critical temperature and the critical density by equating to zero the first and second derivatives of pressure with respect to density. We obtain $n_{\mathrm{c}}=\frac{1}{2}$ and $k_{\mathrm{B}} T_{\mathrm{c}}=$ $z\left(J_{1}+J_{2}\right) / 2$, or $\left(J_{1}+J_{2}\right) / 2 k_{\mathrm{B}} T_{\mathrm{c}}=1 / z$. This is the

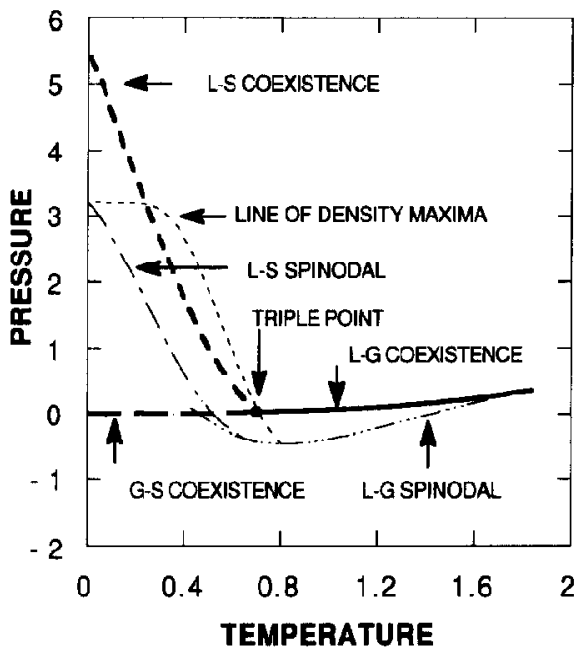

Fig. 2. Coexistence lines, spinodal boundaries of the liquid and the line of density maxima in the liquid phase. Note that the line of density maxima meets the liquid-gas spinodal at the point of reentrance and the liquid-solid spinodal at zero temperature. Both temperature and pressure are expressed in units of $J$, i.e. by fixing $k_{\mathrm{B}}=1$ and the value of the volume to 1 .
Bragg-Williams expression for $T_{c}$, except that in our case the interaction strength $J_{1}$ is temperature dependent. Fig. $1 \mathrm{~b}$ shows $\left(J_{1}+J_{2}\right) / 2 k_{\mathrm{B}} T$ plotted against temperature. Also shown is the value of $1 / z$. Note that $\left(J_{1}+J_{2}\right) / 2 k_{\mathrm{B}} T$ cuts $1 / z$ at two values, implying that the liquid-gas phase separation region is bounded by two critical points, an upper and a lower [4]. The density of the liquid phase is $\frac{1}{2}$ at both critical points, implying that the density must pass through a maximum between the two critical points. Thus, the model displays a density maximum, which is closely connected to the existence of two critical points. Interestingly, both critical points appear at positive pressure, given by $P_{\mathrm{c}}=k_{\mathrm{B}} T_{\mathrm{c}}(\ln 2-0.5)$. Note also that the lower critical point is below $T_{\text {tr }}$, so that the lower critical point is not in the stable region.

The limits of stability of the liquid phase can be obtained by locating the values of $P$ and $T$ where the metastable density value obtained for the liquid phase ceases to be a minimum. To find the limits of stability also for $T<T_{\mathrm{r}}$, we must study the stability of the mean field solution for the liquid phase not only with respect to the gas phase (i.e. along the $n_{\mathrm{A}}=n_{\mathrm{B}}$ line), but also with respect to states with $n_{\mathrm{A}}$ different from $n_{\mathrm{B}}$ (the solid phase). The two solutions for the densities at the spinodal are

$n_{ \pm}=\frac{1}{2}\left[1+\left(1-\frac{2 k_{\mathrm{B}} T}{z\left(J_{2} \pm J_{1}\right)}\right)^{1 / 2}\right]$.

The solution $n_{+}$corresponds to the limit of stability with respect to the gas phase, while the solution $n_{-}$ corresponds to the limit of stability with respect to the solid phase. Using these solutions in the expression for the equilibrium pressure (3), we obtain the corresponding expressions for the spinodal values of pressure. We see from eqs. (3) and (4) that the liquid-gas spinodal pressure is non-monotonic, since $2 k_{\mathrm{B}} T /\left(J_{1}+J_{2}\right)$ is non-monotonic. As discussed above, the non-monotonicity in $2 k_{\mathrm{B}} T /\left(J_{1}+J_{2}\right)$ also leads directly to the existence of the density maximum. Thus, in the model, the presence of density maxima and the reentrance of the spinodal are intimately related.

Fig. 2 shows these two spinodals in the $P-T$ plane. Note that they meet tangentially at $T_{\mathrm{tr}}$. We find that the liquid-gas spinodal is the limit of stability above $T_{\mathrm{tr}}$, while the liquid-solid spinodal is the limit of sta- 
bility below $T_{\text {tr }}$ We see that the liquid-gas spinodal is reentrant and changes non-monotonically with temperature, exhibiting an extreme value at a finite temperature and ending at positive pressure at both ends. To our knowledge, the present model is the first to display a liquid-gas spinodal that extends to positive pressures.

We emphasize that the physically relevant limit of stability at positive pressures (at low $T$ ) is the liquid-solid spinodal. The two spinodals form a continuous locus, but the "critical" properties of these two spinodals are different. The response functions (specific heat, compressibility) diverge at liquid-gas spinodal, while at the liquid-solid spinodal they do not - even though they tend to higher values in the same way, as near a liquid-gas spinodal. This can be understood in terms of the nature of the instabilities at the two spinodals. The metastable liquid phase is a minimum of the grand potential $\Omega$ in the $n_{\mathrm{A}}-n_{\mathrm{B}}$ plane, and at both the spinodals, the curvature of $\Omega$ at this minimum vanishes. At the liquid-gas spinodal, the vanishing second derivative is along the $n_{\mathrm{A}}=n_{\mathrm{B}}$ line. This corresponds to the derivative of the pressure with respect to density approaching zero at the liquid-gas spinodal, resulting in a divergent compressibility. However, at the liquid-solid spinodal, the liquid becomes unstable with respect to "crystal ordering", i.e. the curvature of $\Omega$ vanishes along a direction with $n_{\mathrm{A}}$ different from $n_{\mathrm{B}}$. At the liquid-solid spinodal locus, the derivative of the pressure with respect to density for the liquid phase, which has still to be evaluated along the $n_{\mathrm{A}}=n_{\mathrm{B}}$ line, is different from zero. Thus, the compressibility in our model does not diverge at the liquid-solid spinodal.

Next, we study the locus of points where the density is maximum. Fig. 2 shows the line of density maxima, together with the spinodal lines and the coexistence lines. We see that the end points of the line of density maxima meet the liquid-solid spinodal at the upper end and the liquid-gas spinodal at the reentrance point. This is consistent with the predictions of Speedy and Angell [2] and Debenedetti et al. [3], based on thermodynamic consistency. At the lower end, the slope of the liquid-gas spinodal is changed when the line of density maxima meets the spinodal. At the upper end, the two lines meet at $T=0$ where the liquid-solid spinodal ends. The high pres- sure termination of the line of density maxima is again consistent with the prediction of Speedy and Angell and Debenedetti et al.

We also performed Monte Carlo simulations of the Ising model that corresponds to the present latticegas model in 3D. We find that the simulations agree, in their qualitative trends, with mean field results. The simulations yield qualitatively the same phase diagram obtained from the mean field approximation. As expected, however, the critical point for the ferromagnetic transition is overestimated by the mean field approximation.

To confirm the reentrant behavior and the continuity of the limits of stability of the liquid phase, we performed simulations in the metastable regime for the corresponding Ising model and determined the limits of stability by defining a criterion for the lifetime of the metastable phase. The locus of the limits of stability (or, more accurately, the locus of homogeneous nucleation points) thus obtained is shown in fig. $3 \mathrm{a}$, along with the corresponding mean field solution. It is evident that the limit of stability curve is reentrant, and forms a continuous boundary. We can further distinguish the nature of the limit of stability points by studying the phase to which the systcm decays when these limits are reached. The simulations confirm the statement above that below $T_{\mathrm{tr}}$ the limit of stability is the liquid-solid spinodal, while above $T_{\mathrm{tr}}$ the limit of stability is the liquid-gas spinodal.

In fig. $3 b$ wc show the variation of magnetization with temperature obtained from mean field calculations and Monte Carlo simulations of the 3D model for two different constant field values $h=0$ and $h=0.2$. Figs. $3 \mathrm{c}$ and $3 \mathrm{~d}$ show the magnetic susceptibility at constant field for the same values of $h$. The magnetization and susceptibility of the Ising model correspond to the lattice-gas density and compressibility. Thus, we see that the compressibility is nonmonotonic, increases on cooling, and decreases on compression ( since increasing the magnetic field for the Ising model corresponds to increased pressure in the lattice gas). The model displays a region with negative coefficient of thermal expansion, and we show its variation with $T$ in fig. $4 \mathrm{a}$ at constant pressure, obtained from the mean field calculations. In fig. $4 \mathrm{~b}$, we show constant pressure and constant volume specific heats. 

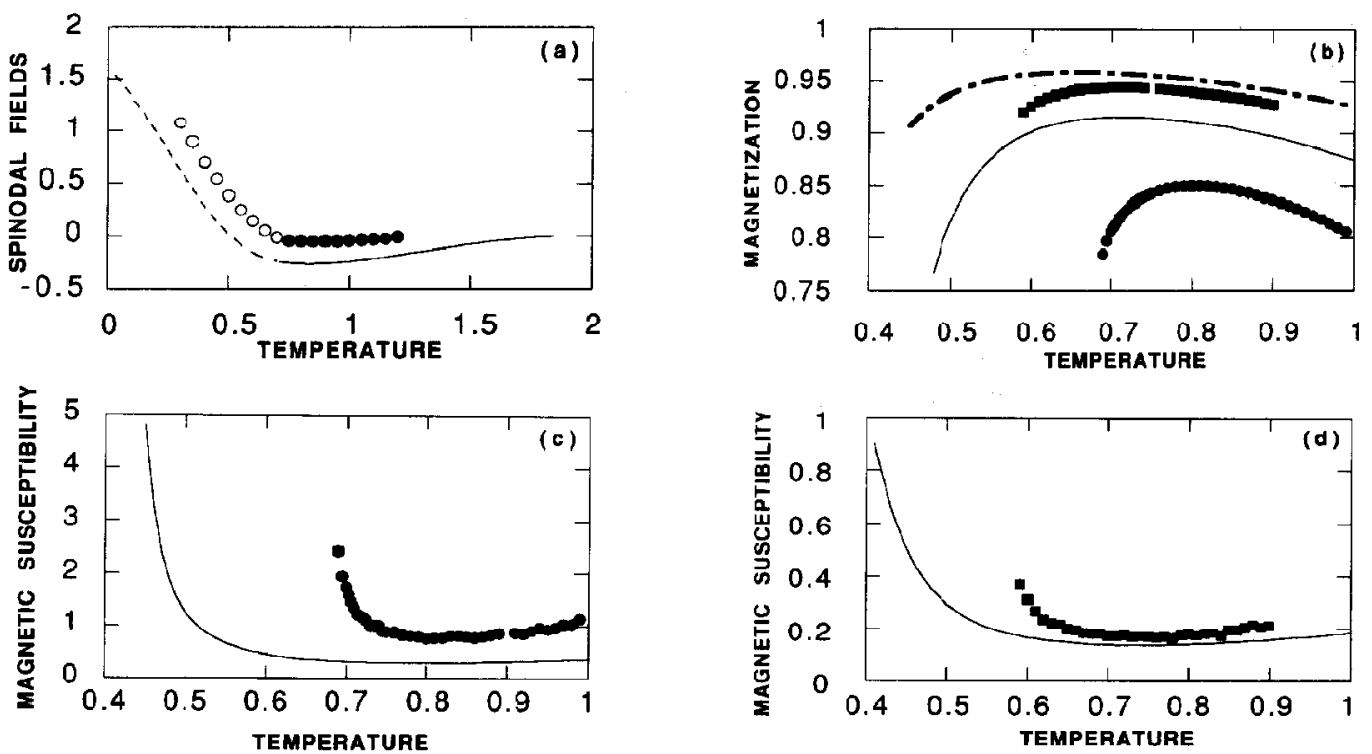

Fig. 3. (a) The liquid-solid (dashed line) and liquid-gas (solid line) spinodal lines from the mean field calculation and homogeneous nucleation points from Monte Carlo simulations (points). Note that the nucleation points "track" the mean field spinodal. Below $T_{\mathrm{tr}}$ the system nucleates to the solid phase (open circles), while above $T_{\mathrm{tr}}$ it nucleates to the gas phase (filled circles). (b) Magnetization at two $h$ values. The continuous lines are mean field results and the data points are from Monte Carlo simulation. The solid line and filled circles are for the magnetic field $h=0$. The dashed line and filled squares are for $h=0.2$. (c) Magnetic susceptibility from the mean field calculation (continuous lines) and Monte Carlo simulations (filled circles) for $h=0$, and (d) $h=0.2$. Note that the magnitude of the susceptibility is lower for the higher $h$ value. Having fixed the value of $k_{\mathrm{B}}$ and of the volume to 1 , the temperature and the magnetic field are expressed in units of $J$, while the magnetization and the magnetic susceptibility are expressed as a pure number and inverse temperature, respectively.

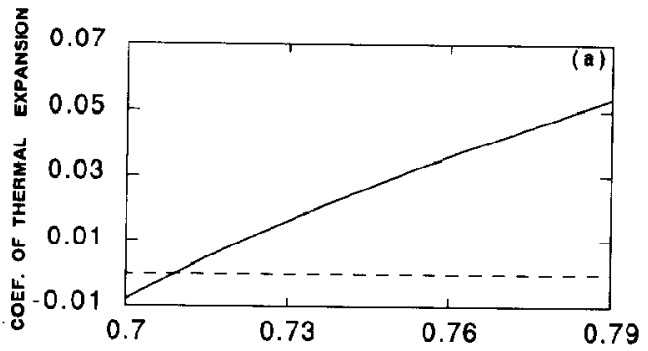

The behavior of the density and the response functions described above are qualitatively similar to the behavior of water, with a phase diagram similar to that of water at low pressures (i.e. the model exhibits only a low density solid phase). The model presented here suggests a supercooling instability for water that is qualitatively different from those where divergences in response functions are predicted [2].

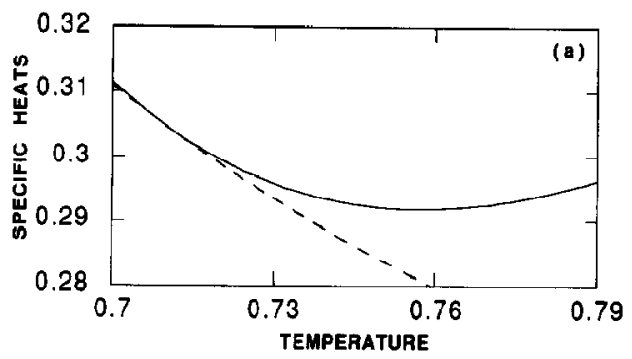

Fig. 4. (a) Constant-pressure coefficient of thermal expansion, which changes sign at the temperature of maximum density, as calculated in mean field approximation. (b) Specific heats at constant pressure and constant volume along a constant pressure trajectory. Note that the specific heats increase upon lowering temperature and, moreover, coincide at the temperature of maximum density. With $k_{\mathrm{B}}=1$, the temperature is expressed in units of $J$, the coefficient of thermal expansion is expressed in inverse temperature units and the specific heat is a pure number. 
We thank A. Angell, P.G. Debenedetti, U. Essmann, M.F. Gyure, H. Larralde, P.H. Poole and R. J. Speedy for helpful discussions, and B. Stovsic for collaboration during the early stages of this research.

\section{References}

[1] C.A. Angell, in Water: a comprehensive treatise, Vol, 7, ed. F. Franks (Plenum Press, New York, 1981) ch. 1; Ann. Rev. Phys. Chem. 34 (1983) 593.
[2] R.J. Speedy and C.A. Angell, J. Chem. Phys. 65 (1976) 851; R.J. Speedy, J. Phys. Chem. 91 (1987) 3354, and references therein.

[3] P.G. Debenedetti and M.C. D'Antonio, J. Chem. Phys. 84 (1986) 3339;

P.G. Debenedetti and M.C. D'Antonio, J. Chem. Phys. 84 (1986) 3339;

P.G. Debenedetti, V.S. Raghaven and S.S. Borick, J. Phys. Chem. 95 (1991) 4540, and references therein.

[4] R.E. Godstein and J.S. Walker, J. Chem. Phys. 78 (1983) 1492 , and references therein.

[5] S. Sastry, F. Sciortino and H,E. Stanley, in preparation. 